\title{
Tricuspid Insufficiency after Laser Lead Extraction
}

\author{
YASSER RODRIGUEZ, M.D., M.B.A., * JULIAN MESA, M.D.,† ERIC ARGUELLES, M.D.,† \\ and ROGER G. CARRILLO, M.D.† \\ From the *Department of Internal Medicine, University of Michigan, Ann Arbor, Michigan; and †Department of \\ Cardiothoracic Surgery, University of Miami Hospital, Miller School of Medicine, Miami, Florida
}

Background: The use of laser lead extraction (LLE) to remove pacemaker and implantable cardiac defibrillator leads has become more prevalent in the past decade. Though the procedure is associated with a low rate of complications, LLE still poses some risks to patients. Some reports have suggested an increase in tricuspid insufficiency (TI) associated with LLE. We present a series of patients who underwent both LLE and complete evaluation for TI with echocardiographic techniques.

Methods: From August 2008 to January 2010, 173 prospective, consecutive patients underwent LLE in a single center. All patients had transesophageal echocardiograms (TEE) during the extraction. Fiftythree patients had tricuspid valve function evaluated a day before the procedure with a transthoracic echocardiogram (TTE), during the procedure with a TEE and 2 days postoperatively with a TTE.

Results: All 173 patients experienced no change in tricuspid valve function during the procedure with TEE. Of the 53 patients who underwent a complete TI evaluation, 38 were males (72\%) and 15 females (38\%), with a mean age of $69.45 \pm 14.08$. Mean ejection fraction was $35.82 \pm 14.72$. Three (6\%) patients experienced TI after the procedure (two mild and one severe, all with tricuspid valve endocarditis); 16 $(30 \%)$ patients were found to have TI before LLE that returned to normal valve function during or after the procedure. Thirty-four (64\%) patients did not experience any significant change of the tricuspid valve performance after LLE.

Conclusion: LLE was not associated with increased TI. (PACE 2013; 36:939-944)

tricuspid insufficiency, pacemaker, artificial, intraoperative complications, implantable defibrillators

\section{Introduction}

The role of implantable cardiac devices continues to increase within medicine. This trend is reflected both by the prevalence of these devices in the population and through the fiscal resources allotted to this area of healthcare. During the 1990s, the number of individuals with pacemakers (PMs) increased by $22 \%$, whereas those with implantable cardiac defibrillators (ICDs) increased 11-fold. ${ }^{1-3}$ During 2006, an estimated 418,000 pacemaker-related procedures $(195,000$ PM implantations and 223,000 PM lead-related procedures) and 114,000 ICD-related procedures were performed in the United States. ${ }^{3}$ Regarding Medicare expenditure data, in the 2009 fiscal year, an estimated $\$ 509,251,000$ was spent on cardiac arrhythmias and conduction disorders. ${ }^{4,5}$

Conflict of Interest: Dr. Carrillo is a consultant for Spectranetics, Sorin ${ }^{\circledR}$, Medtronic ${ }^{\circledR}$, and Sensormatic/TYCO ${ }^{\circledR}$. No other author reports any conflict of interest.

Address for reprints: Yasser Rodriguez, M.D., M.B.A., Department of Cardiothoracic Surgery, 1295 NW $14^{\text {th }}$ Street, Suite H, Miami, FL 33136. Fax: 305-689-2865; e-mail: Rodriguy@med.umich.edu

Received September 26, 2012; revised February 12, 2013; accepted March 15, 2013.

doi: $10.1111 /$ pace. 12160
As the role for devices continues to broaden, it will become increasingly important to expand the literature regarding all aspects of their usage.

The rate of device complications has mirrored the increase in the prevalence of devices within our population. ${ }^{6}$ Because of the fact that these complications usually necessitate device or lead removal, several lead extraction methods have been developed over the past several years-the most recent method being laser-based techniques.

Laser lead extraction (LLE) has been associated with a high rate of procedural success $(96.5 \%)$ and a low level of major complications $(1.4 \%)$ in the setting of experienced operators in high-volume centers ${ }^{7}$; however, some reports have suggested an increase in tricuspid insufficiency (TI) associated with LLE. ${ }^{8,9}$ Our objective is to report our experience with LLE and the development of tricuspid regurgitation (TR).

\section{Patient Population}

\section{Methods}

Data from 173 consecutive patients undergoing LLE at a single high-volume tertiary cardiovascular referral center between August 2008 to January 2010 were obtained from a prospective registry. All patients had transesophageal echocardiograms (TEE) during the extraction. From this population, 53 patients had tricuspid valve

(C)2013, The Authors. Journal compilation (C2013 Wiley Periodicals, Inc. 
function evaluated 1 day before the procedure with a transthoracic echocardiogram (TTE), during the procedure with an intraoperative TEE, and 2 days postoperatively with a TTE. The remaining 120 patients did undergo a pre- and postprocedure TTE; however, the timing of their postprocedure TTE did not occur on day 2 (the timing ranged from day 3 to 8). Informed consent was obtained from each patient, and the study protocol conforms to the ethical guidelines of the 1975 Declaration of Helsinki as reflected in a priori approval by the institution's human research committee.

\section{Echocardiograms}

Both transthoracic and TEE were interpreted by a single certified cardiologist echocardiographer. This echocardiographer was blind to the study. The severity of the TR was categorized as trace, mild, moderate, or severe based on international guidelines. ${ }^{10}$

\section{Definitions}

Extraction was defined in accordance with the Heart Rhythm Society Expert Consensus document from 2009 ${ }^{11}$ : Removal of leads that have been implanted for more than a year or leads that required specialized laser equipment. The indications for transvenous extractions were also defined by the guidelines; examples were infection, chronic pain, thrombosis, venous stenosis, and functional or nonfunctional leads. The infection category was further subdivided and defined: (1) Pocket infection was defined as local signs of inflammation, device, or lead erosion; (2) Device endocarditis was defined according to the modified Duke criteria for endocarditis; (3) Bacteremia where positive blood cultures were the only finding. The terms procedural clinical success, procedure failure, major complications, and minor complications were taken from the aforementioned document.

\section{Laser Extraction}

Laser sheaths (Spectranetics ${ }^{\circledR}$, Colorado Springs, CO, USA) were used in all the cases by a single operator. General anesthesia and intraoperative TEE monitoring were used in all cases. The incision was performed in the infraclavicular space. En bloc encapsulectomy was performed in all the cases. The device was then removed, and the leads were dissected up to the subclavian ligament. A lead locking device was inserted in the inner channel of the multifilar coaxial conductor. Appropriately sized and calibrated laser sheaths were used under fluoroscopic guidance to release the binding sites. An outer sheath was used in approximately $25 \%$ of the cases. As the laser sheath got closer to the myocardial lead tip interface, counter traction was used to free the tip of the lead. Throughout this procedure, gentle tension was maintained to remain coaxial; the lead was never tugged to reduce trauma to the tricuspid apparatus. Fluoroscopy was the imaging modality used to guide the operator; TEE was used exclusively to evaluate for any complications during the procedure. When the tricuspid valve was crossed, laser application was used; there was no mechanical manipulation. In cases involving infections, the wounds were closed primarily with 2-0 nylon and subcutaneous drains were used. This approach was in accordance with Heart Rhythm Society deemed best practices.

\section{Statistical Analyses}

Variable distributions were determined. Continuous variables were expressed as mean values and standard deviations. Categorical variables were expressed as percents. Differences in the proportions involving categorical variables, namely the severity of TI before and after extraction, were analyzed using McNemar's $\chi^{2}$ square test. The level of significance was set at alpha $=0.05$. All analyses were conducted using IBM SPSS Statistics v. 18 (IBM Corp., Armonk, NY, USA).

\section{Patient Characteristics}

\section{Results}

A total of 173 patients underwent device extraction; from this population, 53 patients had a complete TI evaluation that included a preoperative TTE before the procedure, an intraoperative TEE, and a postoperative TTE 2 days after the procedure. Table I provides a summary of several descriptive variables; Fig. 1 shows distribution of patient population and echocardiographic findings.

\section{Indications, Device Properties, and Procedural Approach}

Information regarding the indications for extraction, device characteristics, and lead implant time are provided in Table II. The most common indication was infection, and the mean lead implant time was over 3 years.

\section{Echocardiogram Findings Pre- and Postprocedure}

There were no intraoperative changes in the severity of TI appreciated via TEE in all 53 patients; however, there were changes appreciated when the severity of TI was assessed via preand postoperative TTE. The severity of TI was dichotomized into normal, trace, and mild versus moderate and severe; the difference in the proportion pre- and postprocedure was compared using McNemar's test, and it was found to be statistically significant (without Yates' correction $\mathrm{P}=.003$, 
Table I.

Patient Characteristics $(n=53)$

\begin{tabular}{lc}
\hline Characteristic & Value \\
\hline Age (mean \pm SD) & $69.45(\mathrm{SD} \pm 14.08)$ \\
Gender, $\mathrm{n}(\%)$ & $38(72 \%)$ \\
Male & $15(28 \%)$ \\
Female & \\
Race, $\mathrm{n}(\%)$ & $46(86 \%)$ \\
Caucasian (including Hispanic) & $7(13 \%)$ \\
African American & $35.8( \pm 14.7)$ \\
Ejection fraction* & \\
Comorbid conditions & $46(87 \%)$ \\
Hypertension & $37(69 \%)$ \\
Coronary artery disease & $27(51 \%)$ \\
Diabetes mellitus & $14(26 \%)$ \\
Chronic kidney disease & $10(20 \%)$ \\
Hemodialysis & $53(100 \%)$ \\
CHF, n (\%) & $22(42 \%)$ \\
NYHA I & $15(28 \%)$ \\
NYHA II & $8(15 \%)$ \\
NYHA III & $8(15 \%)$ \\
NYHA IV & \\
\hline
\end{tabular}

*Assessed via preoperative transthoracic echocardiogram.

$\mathrm{CHF}=$ congestive heart failure; NYHA = New York Heart Association; SD = standard deviation.

with Yates' correction $\mathrm{P}=.008$; Table III). Three (6\%) patients experienced worsened or new onset TI after the procedure-all three of these patients had tricuspid valve endocarditis with a valvular vegetation detected by imaging. Sixteen (30\%) patients already displaying different degrees of TI before the procedure experienced an improvement in the severity of TI via postprocedure TTE. Thirty-four $(64 \%)$ patients did not experience any significant change of the tricuspid valve performance after LLE.

Of note, from the 120 patients who did not meet inclusion criteria (because of the fact that their pre- and post-TTEs did not occur on the defined time period), there was no change in their TR severity during the procedure via TEE. There was a significant difference in TR severity in their pre- and post-TTEs. From this group, 66 patients had normal/trace/mild TR before the procedure and 85 after the procedure; 54 patients had moderate/severe TR before the procedure and 35 after (McNemar's $\mathrm{P}<0.01$ ).

\section{Intraoperative Transesophageal Echocardiography}

None of the 173 patients from the prospective registry were observed to have worsening TI via intraoperative TEE (Fig. 1).

\section{Discussion}

Our findings demonstrate that there was no evidence of an increase in the severity of TI using intraoperative TEE; however, there were significant changes appreciated when the severity of TI was assessed via pre- and postoperative TTE. This discrepancy may be explained by confounding variables such as the imaging modality used to assess TR, the presence of valvular involvement in an implantable cardiac device infection, and the patient's fluid status (particularly patients with end-stage renal disease on hemodialysis), among other factors. The method of extraction may greatly influence the development of postextraction TI; the fact that our study used solely LLE to reduce the magnitude of force applied to the tricuspid apparatus likely explains our different experience compared to current literature. In addition, the importance of recognizing tricuspid valve involvement during cardiac device infective endocarditis (CDIE) is imperative given that it portends a worse prognosis.

In our study, we utilized both TTE and TEE. With respect to assessing the severity of TI, both methodologies are comparable, given the fact that even when parasternal and apical views are suboptimal using a TTE, subcostal views allow color flow mapping and recording of hepatic vein pattern. ${ }^{10}$ The main utility in using TEE in our study is that it allowed us to monitor for changes in valvular function intraoperatively in addition to the fact that it may increase the sensitivity for detecting valvular vegetations. ${ }^{12}$ In all 173 cases, including the 53 patients who underwent a complete TI evaluation, there were no changes in the severity of TR appreciated intraoperatively. From the 53 patients, 16 were noted to have an improvement and three had worsening valvular functioning via TTE. This discrepancy between TTE and TEE may be a reflection of confounding variables in the perioperative period. Ten of the 16 patients who demonstrated an improvement in valvular function underwent dialysis immediately following the procedure. Moreover, all three patients who experienced an increase in TR had evidence of tricuspid valve involvement (vegetations) in conjunction with CDIE. Of note, the three patients with endocarditis had newly implanted leads. The mechanism behind why these patients experienced worsening TR following the procedure is unclear.

Our study used solely laser sheath extraction to remove device leads; we did not attempt simple traction nor did we employ any other lead extraction technique. The original PLEXES (Pacing Lead Extraction With the Excimer Sheath) trial, a randomized prospective clinical trial, 


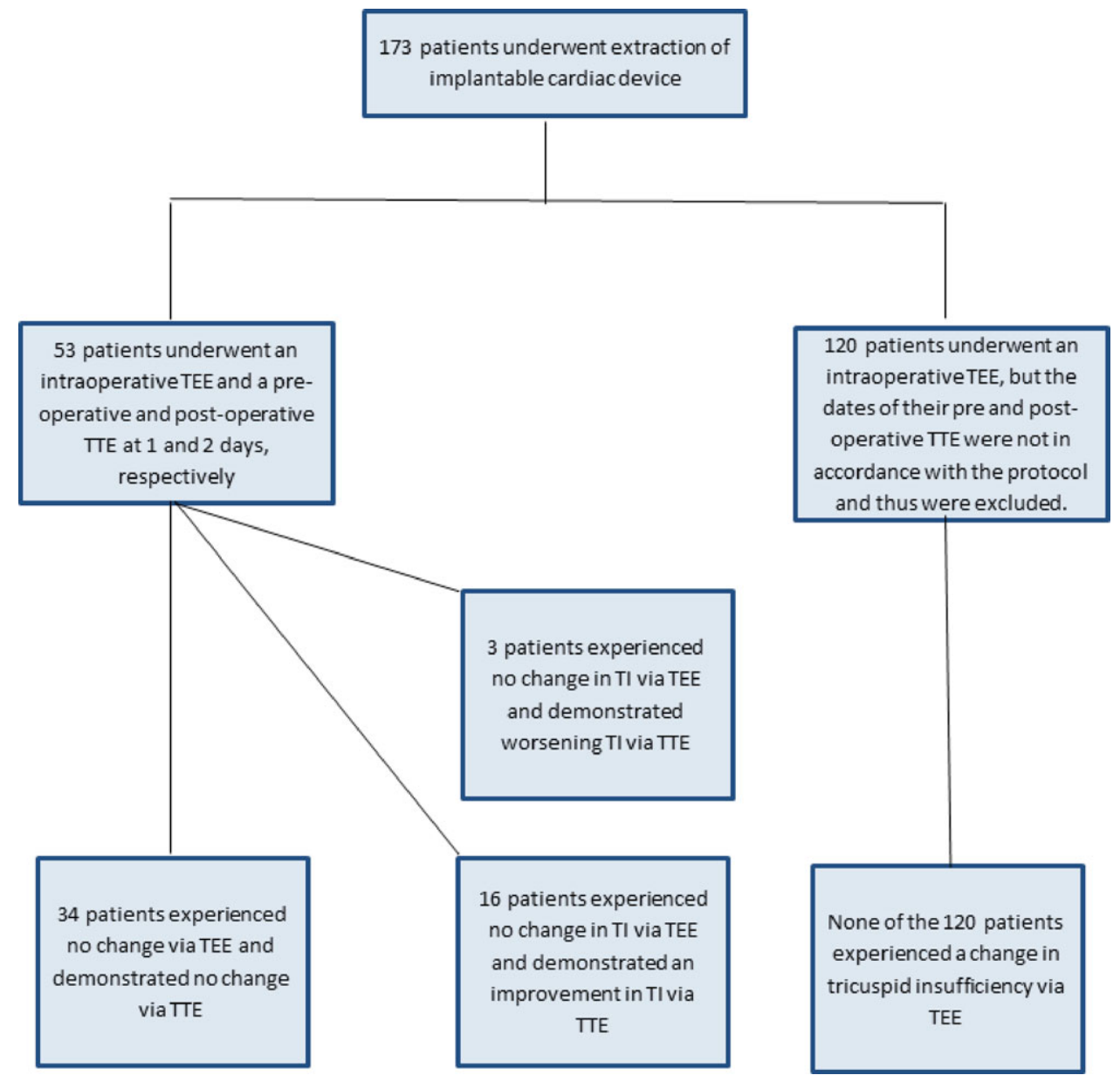

Figure 1. Distribution of patient population and echocardiographic findings. TEE $=$ transesophageal echocardiogram; TTE = transthoracic echocardiogram.

compared a laser sheath to a nonlaser cohort in 301 patients. The procedural success rate and major complication rate in the laser cohort were $94 \%$ and $1.96 \%$, respectively, whereas the success rate for the nonlaser cohort was $64 \%$, often requiring crossover to laser sheath extraction. ${ }^{13}$ The major reason between this difference in procedural success rate may be a function of the fibrotic attachments that gradually encompass the lead. These fibrotic attachments have been described involving the tricuspid valve apparatus and ventricular device leads. ${ }^{14,15}$ Telescoping stainless steel and polymer sheaths require mechanical force to pass them over the leads through dense fibrous tissue, whereas the laser sheath emits pulsed ultraviolet light that ablates the tissue. ${ }^{13}$ Based on the laser sheath's method of action, it likely reduces the magnitude of force applied to the tricuspid apparatus. Our study is the first to assess TI following lead extraction that uses solely LLE in every case without any other technique. This may likely explain the different results that we obtained compared to the current literature. We used gentle tension to stay coxial during the extraction, to further prevent any injury to the SVC. Our complication rate was $1.2 \%$, and we did not experience any injury to the superior vena cava. Our complication rate is in line with the Lexicon study, which was $1.4 \% .^{7}$

Other studies have analyzed the use of laser sheath lead extraction and the development of TR. In a study performed by Franceschi et al., 237 ventricular leads were removed from 208 patients. ${ }^{8}$ The method their group employed for lead extraction was first simple traction or, if the lead had been implanted for more than 6 months, traction after introducing a locking stylet into the lead lumen. Only when removal by simple traction was not successful they employed either laser sheath or lasso (the choice between these two was left to the operator). All patients underwent a preoperative and a postoperative TTE to assess 
Table II.

Indications, Device Properties, and Procedural Approach $(n=53)$

$\begin{array}{lc}\text { Indications, } n(\%) & \\ \text { Infection } & 41(78) \\ \text { Malfunction } & 12(22) \\ \text { Devices, } n(\%) & 20(38) \\ \text { PM } & 24(45) \\ \text { ICD } & 9(17) \\ \text { CRT-ICD } & \\ \text { Leads, } n(\%) & 111 \\ \text { Total } & 40(37) \\ \text { Atrial } & 60(54) \\ \text { Ventricular* } & 11(10) \\ \text { Coronary sinus } & 38.4 \pm 42.8 \\ \text { Lead time (mo.) } & 111(100) \\ \text { Approach, } n(\%) & 146 \pm 23 \text { minutes } \\ \text { Subclavian } & 38 \pm 11 \text { minutes } \\ \text { Total procedure time } & \\ \text { Fluoroscopy Time } & 6(11) \\ \text { Size of sheath used } & 43(81) \\ \text { 12 Fr } & 4(8) \\ \text { 14 Fr } & \end{array}$

CRTICD = cardiac resynchronization therapy implantable cardiac defibrillator; $I C D=$ implantable cardiac defibrillator; $P M=$ pacemaker.

for TR. In their experience, use of laser sheath for lead removal, after failure of simple traction, was the single most powerful risk factor for traumatic tricuspid regurgitation (TTR). The authors stated that the role of laser sheath in the occurrence of TTR was difficult to assess in their study because that modality was reserved for difficult extractions. $^{7}$
Evidence of tricuspid involvement during CDIE portends a worse prognosis when compared to CDIE alone. A recent study by Athan et al., a prospective multicenter study, enrolled 2,760 patients with infective endocarditis. ${ }^{16}$ From this population, 177 were found to have CDIE. Their group compared mortality at 1 month and at 1 year between a cohort with CDIE and no evidence of valvular involvement and a cohort with CDIE and evidence of valvular involvement; of note, tricuspid valve involvement accounted for $65 \%$ of all cases of CDIE with concomitant valvular involvement in their study. The difference in mortality at 1 month for the cohort without evidence of lead vegetation and that with evidence was $79 \%$ and $62 \%$, respectively; the difference in mortality at 1 year for the same cohorts was $50 \%$ and $30 \%$, respectively. ${ }^{16}$ These results emphasize the importance of prompt management following the guidelines established by Baddour et al., emphasizing device explantation and the appropriate use of antibiotics. Moreover, ${ }^{17}$ the results also encourage health practitioners to further stratify patients with CDIE into subgroups, particularly those with evidence of valve involvement, because their prognosis is often worse.

\section{Study Limitations}

The study population consisted of 53 patients; the sample size was limited and originated from a single tertiary referral center and a single operator was used. A large population from several facilities in addition to several operators could be used to increase the generalizability of the findings. In addition, the average lead time in this study was approximately 38 months. A cohort with a greater lead time should be assessed in future studies. Of note regarding our cohort of patients, the patients with structural damage to

Table III.

Echocardiogram Findings Pre- and Postprocedure $(n=53)$

\begin{tabular}{|c|c|c|c|}
\hline & $\begin{array}{c}\text { Preoperative } \\
\text { Echocardiogram }\end{array}$ & $\begin{array}{l}\text { Postoperative } \\
\text { Echocardiogram }\end{array}$ & $\mathbf{P}$ \\
\hline \multicolumn{4}{|c|}{ Tricuspid insufficiency, n (\%) } \\
\hline Normal & $10(19)$ & $20(38)$ & \\
\hline Trace & $8(15)$ & $9(17)$ & \\
\hline Mild & $13(25)$ & $11(21)$ & \\
\hline Moderate & $16(30)$ & $8(15)$ & \\
\hline Severe & $6(11)$ & $5(9)$ & \\
\hline Normal/trace/mild & $31(58)$ & $40(75)$ & $.003^{*}$ \\
\hline Moderate/severe & $22(42)$ & $13(25)$ & \\
\hline
\end{tabular}

*The $\mathrm{P}$ value is derived using an uncorrected McNemar $\chi^{2}$ square. The $\mathrm{P}$ value corrected for continuity (Yates) is 0.008. 
the tricuspid valve that were symptomatic and refractory to medical management were managed surgically as they were initially referred directly for surgical evaluation. The patients who had severe TR and structural damage that were

\section{References}

1. Rodriguez Y, Garisto JD, Carrillo RG. Laser lead extraction in the octogenarian patient. Circ Arrhythm Electrophysiol 2011; 4:719723.

2. Kurtz SM, Ochoa JA, Lau E, Shkolnikov Y, Pavri BB, Frisch D, Greenspon AJ. Implantation trends and profiles for pacemakers and implantable cardioverter defibrillators in the united states: 19932006. Pacing Clin Electrophysiol 2009; 33:705-712.

3. Jones DL, Adams R, Carnethon M, De Simone G, Ferguson TB, Flegal K, Ford E, et al. Heart disease and stroke statistics 2009 update: A report from the American Heart Association Statistics Committee and Stroke Statistics Subcommittee. Circulation 2009; 119:e21-e181.

4. CMS. Medicare ranking for all short-stay hospitals: Fiscal year 2009. https://www.cms.gov/DataCompendium/15_2009_Data_ Compendium.asp (accessed June 22, 2012).

5. CMS. Medicare short-stay hospital MS-DRGs ranked by discharges: Fiscal year 2009. https://www.cms.gov/DataCompendium/ 15_2009_Data_Compendium.asp (accessed June 22, 2012).

6. Voigt A, Shalaby A, Saba S. Continued rise in rates of cardiovascular implantable electronic device infections in the United States: Temporal trends and causative insights. Pacing Clin Electrophysiol 2010; 33:414-420.

7. Wazni O, Epstein LM, Carrillo RG, Love C, Adler SW, Riggio DW, Karim SS, et al. Lead extraction in the contemporary setting: The lexicon study. J Am Coll Cardiol 2010; 55:579-586.

8. Franceschi F, Thuny F, Giorgi R, Sanaa I, Peyrouse E, Assouan X, Prévôt S, et al. Incidence, risk factors, and outcome of traumatic tricuspid regurgitation after percutaneous ventricular lead removal. J Am Coll Cardiol 2009; 53:2168-2174.

9. Glover BM, Watkins S, Mariani JA, Yap S, Asta J, Cusimano RJ, Ralph-Edwards AC, et al. Prevalence of tricuspid regurgitation and pericardial effusions following pacemaker and defibrillator lead extraction. Int J Cardiol 2010; 145:593-594. managed medically were referred to our facility for lead extraction.

\section{Conclusion}

LLE was not associated with increased TI.

10. Zoghbi WA, Enriquez-Sarano M, Foster E, Grayburn PA, Kraft CD, Levine RA, Nihoyannopoulos P, et al. Recommendations for evaluation of the severity of native valvular regurgitation with twodimensional and Doppler echocardiography. J Am Soc Echocardiogr 2003; 16:777-802.

11. Wilkoff BL, Love CJ, Byrd CL, Bongiorni MG, Carrillo RG, Crossley GH 3rd, Epstein LM, et al. Transvenous lead extraction: Heart Rhythm Society expert consensus on facilities, training, indications, and patient management. Heart Rhythm 2009; 6:10851105.

12. Rodriguez Y, Garist J, Carrillo RG. Management of cardiac devicerelated infections: A review of protocol-driven care. Int J Cardiol 2013; 166:55-60.

13. Wilcoff BL, Byrd CL, Love CJ, Hayes DL, Sellers TD, Schaerf R, Parsonnet V, et al. Pacemaker lead extraction with the laser sheath: Results of the Pacing Lead Extraction With the Excimer Sheath (PLEXES) Trial. J Am Coll Cardiol 1999; 33:1671-1676.

14. Novak M, Dvorak P, Kamaryt P, Slana B, Lipoldova J. Autopsy and clinical context in deceased patients with implanted pacemakers and defibrillators: Intracardiac findings near their leads and electrodes. Europace 2009; 11:1510-1516.

15. Candinas R, Duru F, Schneider J, Luscher TF, Stokes K. Postmortem analysis of encapsulation around long-term ventricular endocardial pacing leads. Mayo Clin Proc 1999; 74:120-125.

16. Athan E, Chu VH, Tattevin P, Selton-Suty C, Jones P, Naber C, Miró JM, et al. Clinical characteristics and outcome of infective endocarditis involving implantable cardiac devices. JAMA 2012; 25;307:1727-1735.

17. Baddour LM, Epstein AE, Erickson CC, Knight BP, Levison ME, Lockhart PB, Masoudi FA, et al. Update on cardiovascular implantable electronic device infections and their management: A Scientific statement from the American Heart Association. Circulation 2010; $121 ; 458-477$. 